\title{
A inserção da Física Moderna e Contemporânea em ambientes reais de sala de aula: uma sequência de ensino-aprendizagem sobre a radioatividade ${ }^{+*}$
}

\author{
Carlos Alexandre dos Santos Batista ${ }^{1}$ \\ Doutorando do Programa de Pós-Graduação em \\ Educação Científica e Tecnológica \\ Universidade Federal de Santa Catarina \\ Florianópolis - SC \\ Maxwell Siqueira ${ }^{2}$ \\ Departamento de Ciências Exatas e Tecnológicas \\ Universidade Estadual de Santa Cruz \\ Ilhéus - BA
}

\section{Resumo}

Passado mais de duas décadas de justificativas sobre a inserção da Física Moderna e Contemporânea no Ensino Médio de Física, os desafios, atualmente, voltaram-se para "como”, na prática, inserir seus conteúdos de maneira inovadora em sala de aula. As recentes pesquisas revelam que apesar de um acúmulo significativo da produção acadêmica recente, cujo propósito é auxiliar pedagogicamente os professores, poucas são as propostas fundamentadas teoricamente que buscam investigar como essa inserção acontece. Nesse sentido, apresentamos uma sequência de ensinoaprendizagem sobre o tópico radioatividade, forjada nos pressupostos teórico-metodológicos da Pesquisa Baseada em Projeto (Design-Based Research) e da Sequência de Ensino-Aprendizagem (Teaching-Learning Sequence) que implementada em escolas públicas, produziu conhecimentos relevantes a serem compartilhados com a comunidade do ensino de Física. A proposta permite que professores e pesquisadores possam compreender questões sobre como, quando e por quê, de fato, a inserção da Física Moderna e Contemporânea pode ocorrer de forma não tradicional. Para tanto, a importância desta proposta se revela para o

\footnotetext{
${ }^{+}$Insertion of Contemporary and Modern Physics in classroom: a teaching learning sequence on radioactivity

* Recebido: agosto de 2016. Aceito: maio de 2017.

${ }^{1}$ E-mail: casbatitauesc@gmail.com, ${ }^{2}$ E-mail: maxwell_siqueira@hotmail.com
} 
Ensino Médio de Física à medida que traduz sua capacidade de transformar as demandas teóricas sobre a inovação curricular e metodológica em intervenções práticas de sala de aula. Acrescentamos que a disponibilidade das fontes necessárias para encontrar os planos de aula, questionários, textos, vídeos da sequência de ensino-aprendizagem, mostra a contribuição deste trabalho para professores e pesquisadores e, em especial, para a melhoria da aprendizagem científica dos estudantes na Educação Básica.

Palavras-chave: Física Moderna e Contemporânea; Radioatividade; DBR-TLS.

\begin{abstract}
After more than two decades of justifications on the insertion of Modern and Contemporary Physics in the high school Education, the current challenge regards to how this content can be inserted in the classroom in an interesting and innovative way. Recent research reveals that despite a significant accumulation of recent academic research, whose purpose is to assist teachers pedagogically, few are grounded and proposed theoretically seeking to investigate how this integration happens. In this sense, we present a teaching-learning sequence on the topic of radioactivity, forged in the theoretical and methodological assumptions of Design-Based Research and a Teaching-Learning Sequence that, when implemented in public schools in the south of Bahia, produced the relevant knowledge to be shared with the community on teaching physics. Forged in our assumptions, the proposal allows teachers and researchers to understand questions about how, when and why, in fact, the inclusion of Modern and Contemporary Physics can occur in a non-traditional way. Therefore, the importance of this proposal is revealed to the high school of physics as it translates its ability to transform the theoretical demands on the curriculum and methodological innovation in the practical interventions in the classroom. We add that the availability of the necessary sources to find lesson plans, quizzes, texts, videos of teaching-learning sequence, shows the contribution of this work for teachers and researchers, in particular, to improve the scientific learning of students in the Basic Education.
\end{abstract}

Keywords: Contemporary and Modern Physics; Radioactivity; DBR$T L S$. 


\section{Introdução}

A contínua evolução da Física e a insatisfação com o processo de ensino-aprendizagem de seus conteúdos têm gerado uma demanda crescente para pesquisadores e professores acerca de uma efetiva inovação curricular e metodológica na Educação Básica. Com efeito, a inserção da Física Moderna e Contemporânea (FMC) como linha de investigação estabelecida na área (MONTEIRO; NARDI; BASTOS FILHO, 2012), nas últimas duas décadas, tem sido uma das principais alternativas para alcançar esse empreendimento (GIL et al., 1987; OSTERMANN; MOREIRA, 2000; NETO; CAVALCANTE; OSTERMANN, 2015). Isso porque a sua inserção pode diminuir a defasagem dos conteúdos ensinados e superar, de forma inovadora, as aulas tradicionais, muitas vezes, pautadas no livro didático (DELIZOICOV et al., 2002).

No cenário atual, Pereira e Ostermann (2009), Neto, Cavalcante e Ostermann (2015) revelam que apesar do acúmulo de produções científicas recentes (módulos didáticos, jogos, hipermídias, simulações computacionais, textos didáticos, dentre outros) presentes na literatura, poucos são os estudos fundamentados em referenciais teórico-metodológicos para a sua construção que buscam investigar como a inovação curricular e metodológica acontece, na prática, em sala de aula.

Nesse contexto, apresentamos uma sequência de ensino-aprendizagem sobre o tópico radioatividade, forjada nos pressupostos teórico-metodológicos da Design-Based Research $(D B R)$ - Pesquisa Baseada em Projeto - (COLLECTIVE, 2003) e da Teaching-Learning Sequence (TLS) - Sequência de Ensino-Aprendizagem - (LIJNSE; KLAASSEN, 2004) que foi implementada em salas de aulas da Educação Básica. Vale salientar que, apesar de pouco difundidas no Brasil, as pesquisas fundamentadas nesses referenciais têm sido desenvolvidas por alguns grupos de pesquisa (NICOLAU et al., 2013; BATISTA, 2015).

Para Nicolau et al. (2013), as pesquisas que se propõem trabalhar utilizando os referencias DBR-TLS constituem um movimento consistente de pesquisadores, em níveis internacionais, pois buscam preencher as lacunas e limitações do ensino tradicional, atuando com mais consistência na produção de conhecimentos destinados à Educação Básica.

\section{A Pesquisa Baseada em Projeto e a Sequência de Ensino-Aprendizagem (The Design Based Research and Teaching-Learning Sequence)}

A $D B R-T L S$, como referenciais teórico-metodológicos, surgem das preocupações dos pesquisadores de diversas instituições do mundo em criar um elo coerente entre as demandas teóricas subjacentes ao ensino de Ciências e as práticas educacionais em sala de aula (COLLECTIVE, 2003). Tem como propósito desenhar um objeto de intervenção (sequências de ensino-aprendizagem), a fim de compreender as demandas teóricas (por exemplo, a inovação e atualização curricular) e transformá-las em conhecimentos práticos para a melhoria da aprendizagem científica dos estudantes (COLLECTIVE, 2003). 
Segundo a American Educational Research, as pesquisas baseadas em DBR-TLS possuem cinco características importantes que guiam o desenvolvimento do desenho de intervenção rumo aos dados e respostas de pesquisas mais confiáveis (COLLECTIVE, 2003).

A primeira característica revela que o pesquisador busca, de forma ativa e atenta, estabelecer uma íntima relação entre os objetivos, centrais, do objeto de intervenção com as teorias "protoprincípios de design" e "hipóteses de design", a partir de um olhar focalizado nos parâmetros que delas emergem para o desenho (COLLECTIVE, 2003).

Para Nicolau et al. (2013), os “protoprincípios de design” surgem da própria natureza dos conhecimentos didáticos, pois são produzidos por meio dos parâmetros que guiam as discussões no desenvolvimento e aplicação da sequência de ensino-aprendizagem, durante todo o processo.

Mais especificamente, Nicolau et al. (2013) utilizaram como hipóteses de design para seu projeto de sequência de ensino-aprendizagem sobre relatividade do tempo, as noções de obstáculos epistemológicos de Bachelard (1938) - "dificuldades enfrentadas pelos cientistas durante a produção do conhecimento científico em geral" (NICOLAU et al., 2013, p. 4) - e as noções de obstáculos didático-pedagógicos, proposto por Brousseau (1987; 2013) "dificuldades que normalmente são enfrentadas pelos professores ao implementarem sequências didáticas inovadoras" (ibid. p. 4) - que foram ampliados e adaptados para a FMC no contexto escolar (NICOLAU et al., 2013).

A segunda característica diz respeito aos procedimentos para a validação dos conhecimentos, dentro de um processo cíclico de desenho, aplicação, análise e redesenho. $\mathrm{O}$ intuito é garantir que as modificações ou inclusões de novos parâmetros sejam avaliadas ciclo a ciclo e produzam um conjunto de respostas transferíveis a outros contextos (COLLECTIVE, 2003). Em termos práticos, ela pode se traduzir na busca de repostas para a relação ternária estabelecida pelo professor-saber-estudante, no "Sistema Didático" (CHEVALLARD, 1991), no ambiente real de sala de aula (grifo nosso).

A terceira característica qualifica os resultados obtidos ao compartilhar sua relevância aos demais professores e pesquisadores. Segundo Collins et al. (2004), esse compartilhamento pode ser estabelecido pelo reconhecimento da comunidade do ensino de Ciências da DBR-TLS como uma linha de pesquisa que promove investigações importantes sobre quais variáveis estão envolvidas no processo de ensino-aprendizagem e quais influências educacionais elas exercem sobre o processo.

A quarta característica refere-se ao contexto de aplicação, a fim de que sua singularidade possa render dados expressivos e compatíveis com as demandas do ensino. Todavia, para Tiberghien et al. (2009), não parece, em tudo evidente, que os pesquisadores tenham se preocupado, atualmente, em informar em suas pesquisas as singularidades dos contextos em que elas são realizadas. No entanto, autores como Tiberghien e Broux (1983) e Tiberghien (1996) vêm revelando em suas pesquisas que as singularidades dos contextos de implementação das sequências de ensino-aprendizagem são fundamentais para que, quando 
compartilhados com professores e pesquisadores do ensino de Ciências, estes possam identificar tanto os elementos que tornam possíveis, quanto os que não tornam, a aplicabilidade dessas sequências em seus contextos escolares.

Por fim, a quinta característica recomenda o uso de metodologias que registrem e assegurem uma conexão entre os resultados e as aplicações das sequências de ensinoaprendizagem no contexto de sala de aula (COLLECTIVE, 2003). Mesmo sendo alvo de muitas críticas, Méheut e Psillos (2004) relatam que uma das metodologias mais utilizadas tem sido a Pesquisa-Ação. Isso devido ao pesquisador não se colocar de forma neutra diante das realidades encontradas nos contextos dentro e fora dos âmbitos escolares.

Essa característica permite, por exemplo, justificar a escolha do tópico radioatividade para a elaboração da sequência de ensino-aprendizagem, uma vez que foram encontradas várias condições contextuais, dentro e fora dos âmbitos escolares. Uma delas diz respeito a relação do minério de ferro extraído de jazidas de Urânio (elemento químico naturalmente radioativo) da região de Caetité-BA, com o empreendimento federal da implantação do Complexo Intermodal Porto Sul que vai ligar as regiões Oeste-Leste do país por ferrovias. Outra condição está relacionada à própria grade de conteúdos dos cursos técnicos, que serão detalhados na próxima seção.

Em conformidade com essas características, Collective (2003) argumenta que, ao combinar pesquisa educacional empírica com a teoria, em ambientes de aprendizagem, a DBR-TLS torna-se um referencial teórico-metodológico importante para compreender como, quando e porque as inovações educacionais funcionam na prática. Por conseguinte, um grupo de pesquisadores franceses publicou, em 2004, uma sequência de artigos, condensando diversas investigações de natureza $D B R-T L S$, realizadas a partir dos anos 80 , cuja perspectiva era abordar o ensino de Ciências por tópicos, em detrimento de unidades letivas (NICOLAU et al., 2013). Por exemplo, os sistematizados por Ostermann e Moreira (2000), relatividade, radioatividade, fissão e fusão nuclear, efeito fotoelétrico, estrutura da matéria, condutores, dentre outros.

Utilizando os fundamentos das características da DBR, Lijsen e Klaassen (2004) apresentam como "protoprincípios de design", o modelo de "estrutura didática". Suas premissas estabelecem que uma TLS (definida entre seis e doze aulas) seja fruto do entrelaçamento do desenho e aplicação de um tópico científico específico com duração de algumas semanas, em detrimento de unidades letivas. Nesse sentido, o desenho é orientado por seis fases (LIJSEN; KLAASSEN, 2004). A fase um (1) - evoca um interesse global, ou seja, as concepções prévias dos estudantes sobre um conceito científico específico mediado por um motivo de estudo em questão (implicações sociais, éticas, científicas, tecnológicas, históricas e ambientais). A fase dois (2) - estreita esse motivo global com o conceito a ensinar, no intuito de despertar nos estudantes interesses próprios de aprender novos conhecimentos. A fase três (3) - tem a função de entender o conhecimento dos estudantes, a partir de suas concepções e guiá-los na apropriação do conceito científico. Isso se dar em um processo de sistematização dos novos conheci- 
mentos abordados, buscando sempre a sua conceptualização. A fase quatro (4) - tem a finalidade de permitir que os estudantes, após o processo de sistematização, apliquem os conhecimentos em outras situações. A fase cinco (5) - procura possibilitar uma reflexão nos estudantes após a fase quatro (4), permitindo que eles percebam se os conhecimentos aprendidos fornecem, ou não, subsídios para resolverem situações-problemas, a partir dos contextos das implicações acerca do conhecimento. Por fim, a fase seis (6) - recomenda uma avaliação de cada uma das fases para que seja compreendido em que medida professor e estudantes devem se aprofundar no processo de construção dos novos conhecimentos (LIJSEN; KLAASSEN, 2004).

Como forma de estabelecer a relação entre essas fases, Lijsen e Klaassen (2004) buscam representar a "hipótese de design" sob o termo de "abordagem problematizadora". Suas premissas construtivistas estabelecem que a construção de novos conhecimentos científicos por parte dos estudantes só se torna possível se, durante o processo de ensinoaprendizagem, for levado em consideração os conhecimentos existentes em suas estruturas cognitivas. Essa "hipótese de design" não só expressa a íntima relação entre os objetivos centrais da TLS com as teorias educacionais, como também evidencia que, ao longo das fases, o objetivo central é o estreitamento entre os conhecimentos prévios dos estudantes e o conceito científico que se deseja ensinar. Todavia, isso se dá a partir de um processo dialógico, evidenciado no "Losango Didático" - estrutura teórica da TLS - presente na Fig. 1.

Tramitando no âmbito do "Sistema Didático - relação ternária entre professor-saberestudante" - (CHEVALLARD, 1991), a "dimensão pedagógica" e a "dimensão epistêmica" (Fig. 1) exercem na TLS um papel teórico crucial, pois cristalizam o que, de fato, torna-se importante no processo de ensino-aprendizagem das Ciências. Em outras palavras, o estreitamento entre os conhecimentos prévios dos estudantes (presentes no vértice do "Mundo Vivencial") e o conhecimento científico (vértice oposto) se dá por meio da mediação do professor que direciona as aulas e atividades da $T L S$ visando sempre à aprendizagem significativa.

Para alcançar esse nível de materialização teórica, a $T L S$ é forjada na perspectiva da segunda característica da $D B R$ - validação dos conhecimentos, a partir de um processo cíclico de desenho, aplicação, análise, avaliação, redesenho e nova implementação. Para validar os dados produzidos nesse processo cíclico, Méheut e Psillos (2004) distinguem dois tipos independentes de avaliação, que os pesquisadores lançam mãos. A primeira é chamada de "avaliação interna", cuja realização se promove, por exemplo, pelo processo de evolução conceitual dos estudantes (BENARROCH, 2000; 2001; MORTIMER, 2000). 


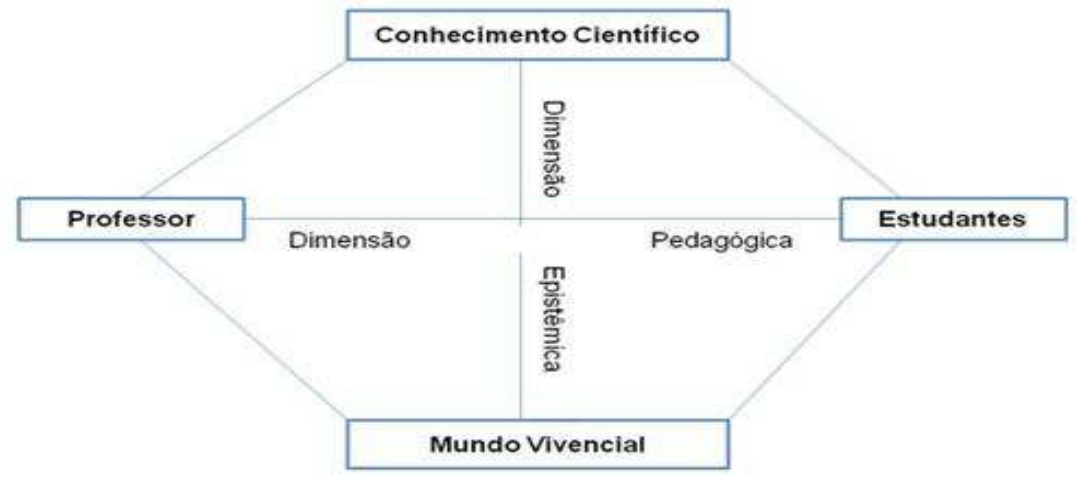

Fig.1 - Estrutura teórica da Teaching-Learning Sequence (MÉHEUT e PSILLOS, 2004, p. 517). (Tradução nossa).

Segundo Benarroch (2000; 2001), a evolução conceitual dos estudantes pode ser investigada em dois planos cognitivos, o plano observável (respostas e esquemas explicativos) e o não observável (esquemas operatórios e específicos), ou seja, respostas verbais, escritas, desenhos e ações exercidas pelos estudantes frente às situações problemas apresentadas (CARMO; SIMONE; MARTORANO, 2010). Nessa perspectiva, os pontos de partida são as concepções prévias dos estudantes - conhecimentos especificamente relevantes, próximos aos aceitos pela comunidade científica, que fazem parte da rede de conhecimentos presentes na estrutura cognitiva do sujeito aprendiz (MOREIRA, 2011) - e suas "ideias informais ou senso comum - forma socialmente construída de descrever e explicar o mundo" (BARRAGAN et al., 2009, p. 3).

Nesse sentido, no início da implementação é utilizado um questionário, cuja finalidade é fazer um mapeamento das concepções prévias dos estudantes. No final, por meio de uma lista de questões, com características bem diferentes do primeiro questionário, ou seja, envolvendo situações-problemas - "estruturas narrativas com lacunas que impõem certas mobilizações cognitivas para o entrevistado” (BARRAGÁN et al., 2009, p. 4), a respeito do conteúdo específico - os estudantes têm a oportunidade de se deparar com questões mais gerais, envolvendo seu cotidiano e o conteúdo, que exigirão deles articulações cognitivas e conceituais, para tomarem suas decisões perante a situação-problema presenciada. Neste momento, tornase possível observar suas respostas sob dois pontos de vista, o primeiro a partir do uso adequado dos conhecimentos científicos, como fundamentos de seus posicionamentos (o que chamamos de indícios de aprendizagem) e o segundo, a partir do uso de ideias de senso comum, que podem representar para determinados grupos de estudantes a existência tanto de falhas e lacunas do processo de ensino-aprendizagem (obstáculos didático-pedagógicos), quanto os próprios obstáculos epistemológicos da aprendizagem.

O outro processo distinguido por Méheut e Psillos (2004) é chamado de "avaliação externa". Diferente da primeira, sua função está diretamente ligada a medir a eficiência de uma 
TLS (sua abordagem construtivista), quando comparada com outros modelos de ensino, tais como o ensino tradicional (MÉHEUT; PSILLOS, 2004). Em nível internacional, esse processo de avaliação é realizado pelos pesquisadores por meio da utilização de grupos controles, turmas de estudantes com a mesma idade e série, também por métodos do "pré-teste e pós-teste" (MÉHEUT; PSILLOS, 2004). A ideia da "avaliação externa" é comparar os resultados obtidos por estudantes que vivenciaram a aplicação da $T L S$, com aqueles que não vivenciaram (MÉHEUT e PSILLOS, 2004), porém necessitando de um número maior de professores e de turmas. Para a realidade do Brasil (principalmente pela escassez de professores de Física em todas as regiões do país), este tipo de avaliação ainda não foi empreendido.

\section{Contextos de implementações}

A sequência de ensino-aprendizagem foi implementada em três turmas de Física de dois Institutos Federais de Educação Ciência e Tecnologia no sul da Bahia. As duas primeiras turmas eram do curso de Segurança do Trabalho (ST11-A, ST12-B) e a terceira, do curso Técnico de Informática (TI).

As justificativas para a escolha desses locais estão relacionadas com a falta de disponibilidade de professores de Física nas escolas da região durante o período da pesquisa e com a própria matriz de conteúdos ensinados, que não contemplam a FMC. Além disso, "certas condições na formação dos professores" consultados não lhes davam segurança (domínio do conteúdo) para trabalhar com a radioatividade em suas aulas.

Nas turmas do curso de Segurança do Trabalho (ST), o professor possuía mestrado em Física Nuclear. No curso de Técnico de Informática (TI), além de uma flexibilidade curricular, o professor estava terminando o mestrado em Educação em Ciências. Com efeito, a primeira implementação ocorreu entre os períodos de maio a julho de 2014 (Fig. 2).

As turmas ST11-A e ST12-B contavam, respectivamente, com vinte e sete e vinte e cinco estudantes. Os estudantes de ST11-A assistiam às aulas das 18h40min às 20h00min, enquanto os de ST12-B assistiam de 20h20min às 22h00min.

O professor (Licenciado em Física e Mestre em Física Nuclear) tinha um encontro com as turmas todas as quintas-feiras. Apesar de ser um instituto Federal, os horários citados nunca eram cumpridos, pois os estudantes tinham problemas com o transporte coletivo, devido ao instituto está localizado fora da zona urbana da cidade.

Importante a ser observado na Fig. 2, é que o tópico radioatividade era contemplado dentro do tema "Proteção Radiológica" abordado em seis encontros de noventa minutos (duas aulas). Contudo, vemos que no decorrer da implementação houve um recesso prolongado (12/06 a 19/16 de 2014) que, juntamente com o problema da chegada e saída dos estudantes em sala de aula, influenciou muito no desenvolvimento da sequência de ensino-aprendizagem (não cumprimento do cronograma).

Na segunda implementação, a turma do curso Técnico de Informática, com vinte e cinco estudantes, não continha uma definição explicita do conteúdo, todavia, o professor 
(Licenciado em Física, terminando o Mestrado em Educação em Ciências) achou a proposta pertinente, visto que os estudantes já tinham tido contato com o conteúdo na disciplina de Química Geral e seria interessante que tivessem uma visão a partir da Física também. A sequência foi implementada no primeiro semestre de 2015, no mês de abril, todas as segundas e quintas-feiras, entre as $10 \mathrm{~h} 10 \mathrm{~min}$ e $11 \mathrm{~h} 50 \mathrm{~min}$ da manhã, durante quatro encontros de (100) cem minutos.

\begin{tabular}{|c|c|c|}
\hline $\begin{array}{c}\text { Datas dos Encontros } \\
\text { Em 2014 }\end{array}$ & $\begin{array}{c}\text { Aulas por } \\
\text { Encontro }\end{array}$ & Conteúdo \\
\cline { 1 - 2 } $08 / 05$ & 2 & \multirow{2}{*}{ Radiação e Proteção Radiológica - Proposta } \\
Interdisciplinar.
\end{tabular}

Fig. 2 - Recorte do cronograma geral da disciplina Física Aplicada. (BATISTA, 2015)

Essas principais características dos contextos são vistas por Tiberghien (1996) tanto como premissas importantes a serem incluídas no processo de avaliação da sequência de ensinoaprendizagem (segunda características da DBR-TLS), quanto compartilhadas com a comunidade do ensino de Física (TIBERGHIEN et al., 2009). O intuito é permitir que esses elementos garantam uma melhor visão e entendimento dos professores quando delas fizerem uso em seus contextos escolares. Para os autores, quanto mais os contextos de implementação das TLS forem compartilhados, mais enriquecedora vai se tornando para o ensino de Ciências, visto que uma das grandes dificuldades desse tipo de pesquisa é que, muitas vezes, ela tem ficado restrita a grupos de pesquisas (COLLECTIVE, 2003; MÉHEUT; PSILLOS, 2004; TIBERGHIEN et al., 2009). 


\section{Conhecimentos produzidos com a primeira implementação}

O desenho piloto da sequência de ensino-aprendizagem sobre a radioatividade foi forjada a partir do modelo de "estrutura didática" e desenvolvida nos pressupostos da "abordagem problematizadora" (LIJSEN; KLAASSEN, 2004). Portanto, foi constituída por doze (12) aulas (Fig. 3) e divididas em seis encontros de noventa minutos, especificamente para atender o primeiro contexto de implementação (Fig. 2).

\begin{tabular}{|c|c|c|c|}
\hline Aulas & $\begin{array}{l}\text { Tempo } \\
\text { (min.) }\end{array}$ & Conteúdo & Recursos de ensino \\
\hline 1 e 2 & 90 & $\begin{array}{l}\text { Implicações da Radioatividade nos contextos } \\
\text { sociais, políticos e econômicos. }\end{array}$ & Vídeos/Questionário. \\
\hline 3 e 4 & 90 & A descoberta da Radioatividade & Vídeo - Texto 1. \\
\hline 5 e 6 & 90 & $\begin{array}{l}\text { Conceitos físicos: força nuclear, } \\
\text { transmutação nuclear e decaimentos alfa, } \\
\text { beta e gama. }\end{array}$ & $\begin{array}{c}\text { Atividade lúdica com bolinhas de isopor } \\
- \text { Texto } 2 .\end{array}$ \\
\hline 7 e 8 & 90 & $\begin{array}{l}\text { Forças nucleares, decaimentos radioativos e } \\
\text { meia-vida. }\end{array}$ & Exercícios. \\
\hline 9 e 10 & 90 & Decaimentos radioativos alfa e beta. & Atividade lúdica - Diagrama radioativo. \\
\hline 11 e 12 & 90 & $\begin{array}{l}\text { Aspectos principais da radioatividade e suas } \\
\text { implicações sociais, políticas e econômicas. }\end{array}$ & $\begin{array}{c}\text { Debate em sala - Texto produzido pelos } \\
\text { alunos. }\end{array}$ \\
\hline
\end{tabular}

Fig. 3 - Desenho da sequência de ensino-aprendizagem sobre a radioatividade. (BATISTA, 2015)

Para a nossa "hipótese de design", presumíamos, assim como Lijsen e Klaassen (2004), que os estudantes de Física do curso técnico de Segurança do Trabalho só poderiam vivenciar uma experiência significativa de ensino-aprendizagem sobre a radioatividade, se considerássemos seus conhecimentos prévios como essenciais para o desenvolvimento das aulas e das atividades, no contexto da "abordagem problematizadora" (LIJSEN; KLAASSEN, 2004; MOREIRA, 2011).

Como forma de materializar essas concepções prévias, achamos conveniente citar alguns exemplos sobre o que os estudantes entendiam por radiação, a fim de atender os pressupostos da fase (1) - levantamento das concepções prévias. Para o estudante da turma ST11 (E5-A), "radiação é uma forma de energia extraída de elementos naturais, ou manipulada, que pode trazer tanto benefícios quanto malefícios à humanidade”. Para ST11 
(E19-A), "radiação são ondas eletromagnéticas ou partículas carregadas com bastante energia, essas ondas podem ser ionizantes, no caso de elementos radioativos; as ondas de telefonia celular, TV e rádio não são consideradas ionizantes". Para o estudante da turma ST12 (E9-B), "radiação é a emissão de energia por raios eletromagnéticos que têm relação com elementos químicos como urânio e Césio". Por fim, ST12 ( $\left.\mathrm{E}_{11}-\mathrm{B}\right)$, "radiação são ondas eletromagnéticas que atingem um corpo ou a mão, podendo contaminar e, consequentemente, desencadear numerosas doenças graves".

Como explicitamos anteriormente, essas concepções prévias são diferentes de senso comum, pois estão muito próximas do conceito de radiação aceito pela comunidade científica, como pode ser observado na definição de Okuno (1988, p. 12): "radiação é uma forma de energia emitida por uma fonte, que se propaga de um ponto a outro sob a forma de partículas com ou sem carga elétrica, ou ainda sob a forma de ondas eletromagnéticas".

Além desse levantamento das concepções prévias, foram trabalhadas as questões científicas, tecnológicas e sociais (CTS) acerca do conteúdo da radioatividade e sua relação com empreendimento federal do Complexo Intermodal Porto Sul, a partir de vídeos curtos ${ }^{2}$ envolvendo enriquecimento de urânio e suas aplicações na medicina, na produção de energia elétrica, na agricultura, dentre outros setores, bem como questões envolvendo os acidentes de Goiânia-GO e o da mina de Caetité-BA.

O segundo encontro (aulas 3 e 4) foi marcado pela busca das ações da fase (2) ampliação das concepções prévias dos estudantes. Para isso, os estudantes fizeram a leitura do Texto 1 - A descoberta da Radioatividade -, produzido por um grupo de pesquisa em inovação curricular (SIQUEIRA, 2006). Juntamente com o professor, foi promovida uma discussão conceitual das questões do texto e do vídeo sobre a descoberta da radioatividade ${ }^{3}$. O objetivo central era permitir uma visão contextualizada sobre a descoberta da radioatividade (caráter humano e colaborativo do trabalho científico), conhecer um pouco sobre os cientistas envolvidos nesse contexto e sistematizar conceitos físicos como fluorescência, fosforescência, emissão e absorção e transmutação nuclear.

Para colocar em prática a fase (3) - estreitamento entre as concepções prévias e os conhecimentos cientificamente aceitos - o professor, iniciando no terceiro encontro (aulas 5 e 6) e finalizando no quarto (aulas 7 e 8), sistematizou os conceitos de transmutação nuclear, força eletromagnética, força nuclear forte e fraca, radiação alfa (núcleos de hélio), radiação beta (elétron ou pósitron) e radiação gama (ondas eletromagnéticas). Para isso utilizou a leitura do Texto 2 - Entra em cena uma nova figura, Ernest Rutherford - (SIQUEIRA, 2006) e a atividade lúdica com bolinhas de isopor (Fig. 4), adaptada de Pietrocola et al. (2010). Para finalizar essa sistematização conceitual, o professor também utilizou de resoluções de exercícios presentes

\footnotetext{
${ }^{2}$ Disponível em: <https://www.youtube.com/watch?v=63gBrF1I_hM $>$; $<$ https://www.youtube.com/watch?v=63UWTcXDdp; https://www.youtube.com/watch?v=X-C3eU_8T5w>. Acesso em: 05 mai. 2016.

${ }^{3}$ Disponível em: <www.youtube.com/watch?v=5VvjBz-jbVc>. Acesso em: 20 mar. 2016.
} 
nos livros didáticos de Física aprovados pelo Plano Nacional do Livro Didático 2012. Vale ressaltar que, por mais que seja evidente a quantidade de conceitos trabalhados nesses dois encontros (180 min.), eles são necessários para que os estudantes desenvolvam uma visão mais ampla da dimensão conceitual da radioatividade. Neste sentido, as atividades lúdicas (Fig. 4 e 5) exercem um papel importante, pois tornam a abordagem dos conceitos citados, mais didático pedagogicamente, não centrado apenas no professor, mas também na participação ativa dos estudantes no processo de ensino-aprendizagem.

$\mathrm{Na}$ atividade da instabilidade nuclear (Fig. 4), por exemplo, as bolinhas, de maneira grosseira, representavam o "núcleo atômico". Os estudantes, munidos de um roteiro e materiais didáticos (bolinhas de isopor, fita adesiva e tesoura), iniciavam a atividade. Em grupo, eles precisavam colar o máximo de bolinhas, antes que a primeira viesse a cair. Quando as bolinhas começavam a cair, precisavam responder os motivos da queda. É importante registrar que a realização da atividade deve ser conduzida desvinculada dos conceitos científicos citados na Fig. 3, aulas (5 e 6), pois as bolinhas não são as partículas.

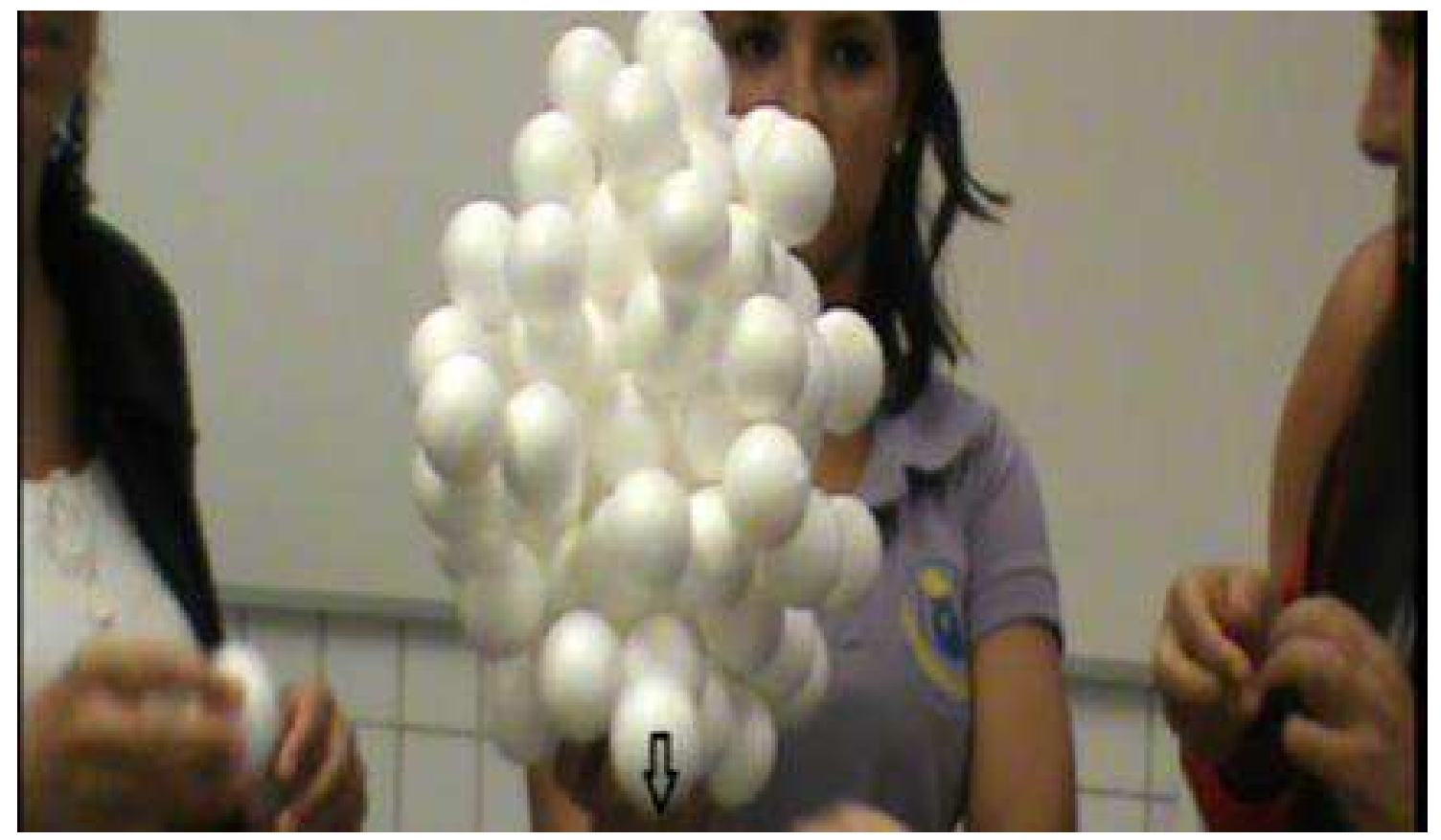

Fig. 4 - Estudantes da turma (ST12-B) realizando a atividade das bolinhas de isopor. (BATISTA, 2015)

Nas aulas (7 e 8), o professor com muito cuidado fazia analogias possíveis entre os conceitos científicos (força nuclear forte, fraca e decaimento radioativo alfa e beta), por exemplo, relacionando a "aderência da fita adesiva" à força nuclear forte. A ideia era permitir que os estudantes não criassem obstáculos de aprendizagem, nem falsa compreensão sobre a geometria do núcleo atômico (função matemática complexa, informada pelo professor a título de conhecimento dos estudantes). Utilizando exercícios, o professor, como mediador do 
processo de ensino-aprendizagem, tentava proporcionar uma maior clareza sobre a ideia da atividade e dos conceitos físicos que a circundavam.

O quinto encontro (aula 9 e 10), cujo momento representou a fase (4 e 5)- aplicação do conhecimento e reflexão dos estudantes, permitiu que os estudantes aplicassem os novos conhecimentos, utilizando outra atividade adaptada (PIETROCOLA et al., 2010), a qual chamamos de "diagrama radioativo" (Fig. 5) e refletissem, por meio de um relatório (Figura 6), se os conhecimentos adquiridos eram suficientes para resolver a atividade ou não.

A atividade da Fig. 5 consistia em uma sucessão de transmutações nucleares (mudança na estrutura atômica do elemento químico radioativo) durante a emissão de partículas alfa (núcleos do elemento químico hélio) ou partículas beta (elétrons ou pósitron). Estatisticamente, essa instabilidade nuclear ocorre pela diferença expressiva entre o número de prótons e o número de nêutrons, além de expressivo número de massa.

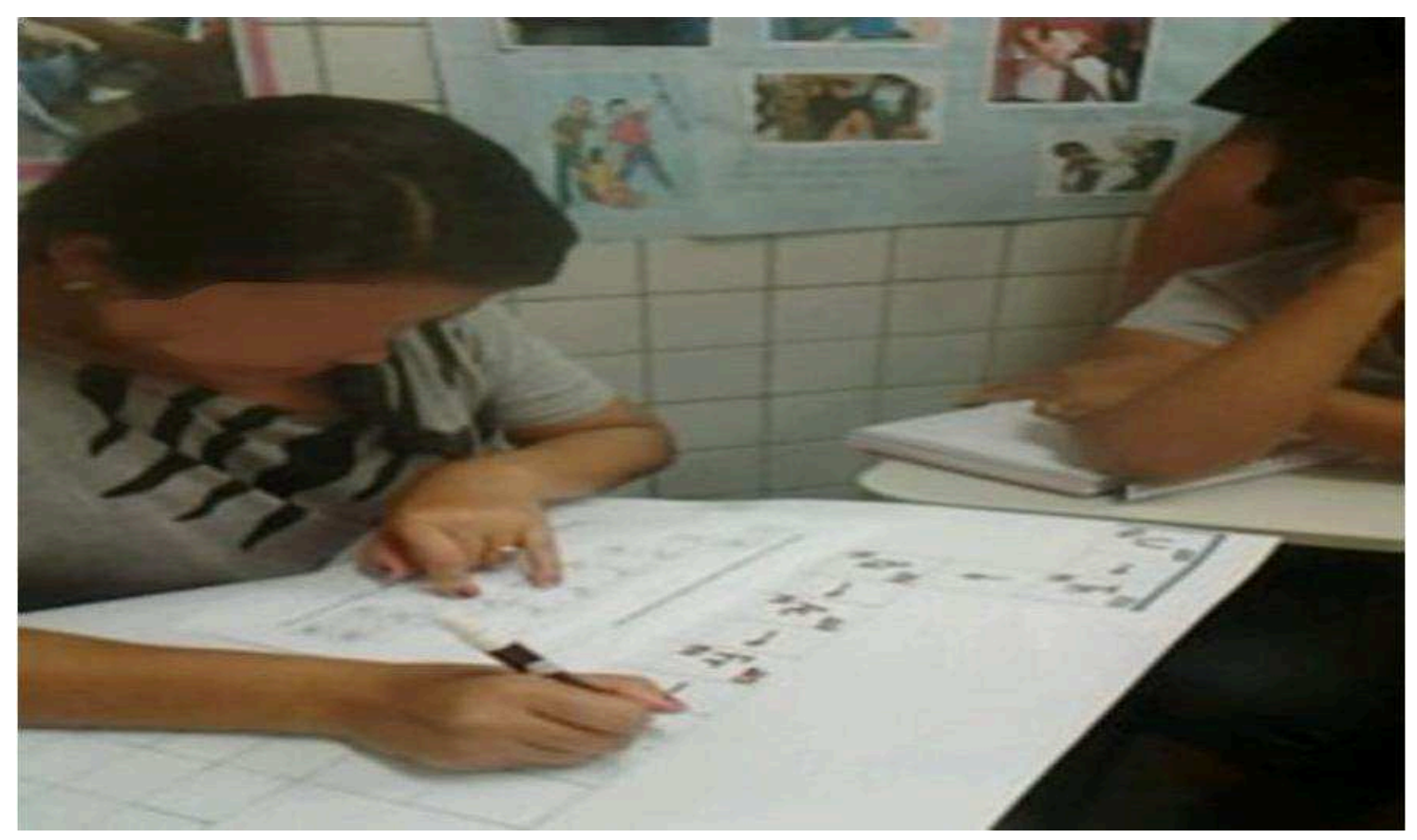

Fig. 5 - Estudantes da turma ST11-A montando o diagrama radioativo. (BATISTA, 2015)

Essa sucessão de transmutações inicia-se com um elemento que pode ser denominado de "núcleo pai" (Urânio ou Tório, por exemplo), pois, ao sofrer transmutação, vai se tornar um novo elemento, também radioativo, até tornar-se um elemento não mais radioativo. Nesta atividade, os estudantes, em grupos, identificavam qual partícula era emitida (alfa ou beta) e quais elementos químicos surgiam a partir dessa emissão.

Nesse sentido, precisavam utilizar os conceitos de transmutação nuclear, de instabilidade nuclear, força forte e força fraca, para explicar conceitualmente tanto o fenômeno da radioatividade, quanto os motivos das emissões das partículas. Os resultados da atividade, 
perante a fase (5), foram satisfatórios em alguns grupos, mas em outros não. Como podemos ver na Fig. 6, os estudantes conseguiram utilizar os conceitos de forma correta. Já para outros grupos, houve tanto o uso incorreto dos conceitos, quanto nenhuma menção dos conceitos estudados na confecção do relatório.

Obs.: Nesta folha, façam um relatório abordando cada etapa realizada. Para o relatório ficar completo, não deixe de falar sobre os conceitos utilizados para justificar a tomada de decisão em cada etapa da atividade.

Ao realizar o estudo sobre decaimento radioativo, observamos que através da transmutação nuclear o elemento " $U$ " sofre transmutações no interior do núcleo. Isso ocorreu a partir da emissão de partículas $\alpha$ (alfa) e $\beta$ (beta), gerando o decaimento gradual, formando um elemento diferente do original.

Esse resultado foi obtido a partir do cálculo usado para identificar tais transmutações, onde:

- Na emissão de partículas $\beta$ (beta), a massa permanece a mesma, enquanto que o número atomico aumentou (1), no entanto pode ser $\beta(-)$ (beta menos) ou $\beta^{(+)}$(beta mais);

- Na emissão de partículas a (alfa), a massa decai em quatro (4), enquanto que o número atomico diminui em dois (2).

Dessa forma, o elemento que inicialmente era urânio, através das transformações sofridas, originou o chumbo. Assim, finalizando o estudo.

Fig. 6 - Relatório apresentado pelos estudantes da turma (ST12-B). (BATISTA, 2015)

Para a fase (6) - reflexão sobre os objetivos alcançados pelas aulas e atividades devido à existência de questões inerentes ao contexto de implementação (Fig. 2) - (feriado, reposição de aula, final de semestre), o sexto encontro não aconteceu. Visto isso, o professor utilizou o encontro para suprir as necessidades apresentadas pelos estudantes na volta às aulas, após o recesso de junho-julho.

Com efeito, a fase seis foi desenvolvida por nós, pesquisadores, ao proceder com uma avaliação rigorosa e sistemática das atividades, dos objetivos que foram alcançados e os que não foram, da necessidade de mais fundamentações teóricas nas atividades e reestruturação das aulas a partir de mudanças estruturais nas abordagens das atividades lúdicas. Essas ações, como explicitamos na seção (II), estão previstas na segunda característica da $D B R-T L S$ e fazem parte da produção dos conhecimentos transferidos para o outro contexto de implementação (curso Técnico de Informática), a partir da inclusão dos novos parâmetros. Todavia, as implicações dessas questões singulares no primeiro contexto são previstas também por outros pesquisadores (LOPES et al., 2008; TIBERGHIEN et al., 2009).

Após um processo rigoroso de avaliação das aulas e das atividades por meio de fundamentações teóricas presentes na literatura (MARTINS, 1990; BANARROCH; 2000; 2001; BARRAGAN et al., 2009; SANTOS; PEREZ-ESTEBAN, 2012), foi possível encontrar indícios e lacunas do processo de ensino-aprendizagem sobre os conceitos da radioatividade abordados (LEACH; SCOTT, 2002), incluir os novos parâmetros (notícias de jornais) 
(SANTOS; PEREZ-ESTEBAN, 2012), bem como reestruturar as principais atividades da sequência ("instabilidade nuclear" e "diagrama radioativo"). O resultado do processo culminou no redesenho da nova sequência de ensino-aprendizagem sintetizada na Fig. 7.

\begin{tabular}{|c|c|c|c|}
\hline Aulas & Tempo (min.) & Conteúdo & Recursos de Ensino \\
\hline 1 & 50 & $\begin{array}{l}\text { Implicações da radioatividade os contextos } \\
\text { sociais políticos e econômicos }\end{array}$ & Vídeos - Questionário 1. \\
\hline 2 & 50 & A descoberta da Radioatividade. & Vídeo - Texto 1. \\
\hline 3 & 50 & $\begin{array}{l}\text { Por que alguns elementos químicos são } \\
\text { radioativos e outros não? }\end{array}$ & Notícias de Jornais - Texto 2. \\
\hline 4 & 50 & $\begin{array}{c}\text { Força nuclear forte, decaimentos radioativos } \\
\text { alfa, beta, gama e meia-vida. }\end{array}$ & Exercícios. \\
\hline 5 & 50 & Instabilidade e transmutação nuclear. & $\begin{array}{l}\text { Atividade lúdica com bolinhas de } \\
\text { isopor. }\end{array}$ \\
\hline 6 & 50 & Instabilidade e transmutação nuclear. & Aula expositiva. \\
\hline 7 & 50 & $\begin{array}{l}\text { Aplicações dos conceitos de decaimentos } \\
\text { alfa e beta e de transmutação nuclear. }\end{array}$ & $\begin{array}{c}\text { Atividade lúdica - "Quebra cabeça } \\
\text { radioativo". }\end{array}$ \\
\hline 8 & 50 & $\begin{array}{l}\text { Avaliação final da Sequência de Ensino- } \\
\text { Aprendizagem. }\end{array}$ & Questionário 2. \\
\hline
\end{tabular}

Fig. 7 - Sequência de ensino-aprendizagem reestruturada. (BATISTA, 2015)

Na Fig. 7, os destaques dessa reestruturação são dados pelas mudanças nas abordagens das atividades, como veremos a seguir, da ampliação do questionário 1 e da elaboração do questionário 2, que serviram como parâmetros para a "avaliação interna" da sequência de ensino-aprendizagem. Um exemplo concreto dessas mudanças ocorreu na abordagem da atividade lúdica das bolinhas de isopor "instabilidade nuclear" e na atividade do "diagrama radioativo", que passou a ser chamada de "quebra-cabeça radioativa".

\section{Conhecimentos produzidos com a segunda implementação}

Depois de avaliada e reestruturada, o redesenho da sequência de ensino-aprendizagem (Fig. 7), passou a contar com apenas oito aulas de cinquenta (50) minutos, visando, principalmente, atender o cronograma das aulas do curso Técnico de Informática (TI).

Nesse sentido, como novo parâmetro incluído na primeira aula, aumentamos o número de questões do questionário (dados da pesquisa) para a sondagem das concepções prévias dos estudantes, utilizando os trabalhos de Martins (1990) e Barragán et al. (2009). Todavia, para a mesma questão, por exemplo, - O que é radiação? - os estudantes do curso de TI apresentaram as mesmas concepções que os estudantes de Segurança do trabalho ST11-A e ST12-B. Por exemplo, Estudante (E3-TI) - "Radiação nos dá a ideia de rádio, que lembra ondas, 
infravermelho, raios-x e outros". Estudante (E14-TI) - "Uma forma de energia eficiente e necessária para a humanidade (não lembro a definição)". Estudante (E20-TI) - "Radiação é uma forma de transferência de energia de um elemento para outro”. Estudante (E22-TI) "São ondas eletromagnéticas que atravessam um ser ou um objeto".

O compartilhamento desse resultado torna-se relevante, pois revela a existência de um padrão de ideias sobre o estudo da radioatividade entre estudantes dos cursos técnicos em diferentes contextos (BARRAGÁN et al., 2009). Com base na literatura, está em comum acordo com diversos estudos realizados em diferentes partes do mundo sobre o assunto (YANG; ANDERSON, 2003; WU; TSAI, 2007).

Na segunda aula (fase 2) não houve inclusão de novos parâmetros, o professor usou os mesmos vídeos e textos sobre a descoberta da radioatividade para construir com os estudantes a mesma cronologia histórica e científica utilizada pelo professor na primeira implementação. A ideia era permitir que os estudantes de TI também pudessem conhecer os aspectos da natureza da Ciência e do trabalho científico por intermédio dos seus personagens.

$\mathrm{Na}$ terceira aula (fase 3), a pergunta - Porque alguns elementos são radioativos $e$ outros não? (MARTINS, 1990) - foi a base da inclusão do novo parâmetro (notícias de jornais) (SANTOS e PEREZ-ESTEBAN, 2012) na sequência de ensino-aprendizagem. O professor contou com o caso verídico da morte por envenenamento por polônio-210 de um ex-agente russo em 2006, Londres - Inglaterra (SANTOS; PEREZ-ESTEBAN, 2012), com o caso do acidente de Fukushima em 2011, no Japão, e com a reportagem dos trinta e cinco anos do acidente de Goiânia - Brasil, lembrado em 2012 pela Secretaria de Saúde do Estado de Goiás (dados da Pesquisa). Em uma abordagem com leituras dos textos utilizados na primeira implementação, o professor tanto sistematizou os conceitos de transmutação nuclear, decaimentos radioativos alfa, beta e gama, isótopos (elementos com mesmo número de prótons) e isóbaros (mesmo número de massa), quanto os efeitos da radiação alfa nos seres vivos.

Na quarta aula, também não houve inclusão de novos parâmetros. O professor trabalhou os conceitos de força nuclear forte, fraca, eletromagnética, transmutação nuclear, decaimentos radioativos alfa, beta e gama com aula expositiva e resolvendo exercícios em sala. Esses vários conceitos trabalhados, apesar de não ser o foco da sequência de ensinoaprendizagem, tornam-se de fundamental importância para que os estudantes de TI também compreendessem a dimensão e profundidade conceitual do tópico radioatividade.

$\mathrm{Na}$ aula cinco, a atividade da instabilidade nuclear teve uma mudança estrutural significativa: os estudantes, em grupo, recebiam um roteiro simples, apenas com questões sem cunhos conceituais, uma quantidade de bolinhas de isopor e fita adesiva. Com esses materiais, iniciavam a atividade, colando uma bolinha nas outras até ver quem conseguia colar uma maior quantidade, antes da primeira bolinha cair (Fig. 8).

A ideia era que eles pensassem quais os motivos que levavam as bolinhas a caírem. $\mathrm{O}$ que acontecia com as mesmas, quando a colagem ultrapassava uma quantidade considerável? Quem era responsável pela queda das bolinhas (peso das bolinhas em relação à aderência da 
fita). Na aula seis, o professor retomou os conceitos trabalhados na aula quatro e, com base nas respostas dos estudantes às perguntas do roteiro, introduzia as novas questões, por exemplo: Que analogia pode ser feita com as bolinhas, em relação ao núcleo do átomo? Que analogia pode ser feita por meio da queda das bolinhas para entender o fenômeno de transmutação nuclear? Com isso, buscava fazer as relações conceituais necessárias para que os estudantes pudessem estreitar ainda mais suas concepções prévias com os conhecimentos da radioatividade ensinados.

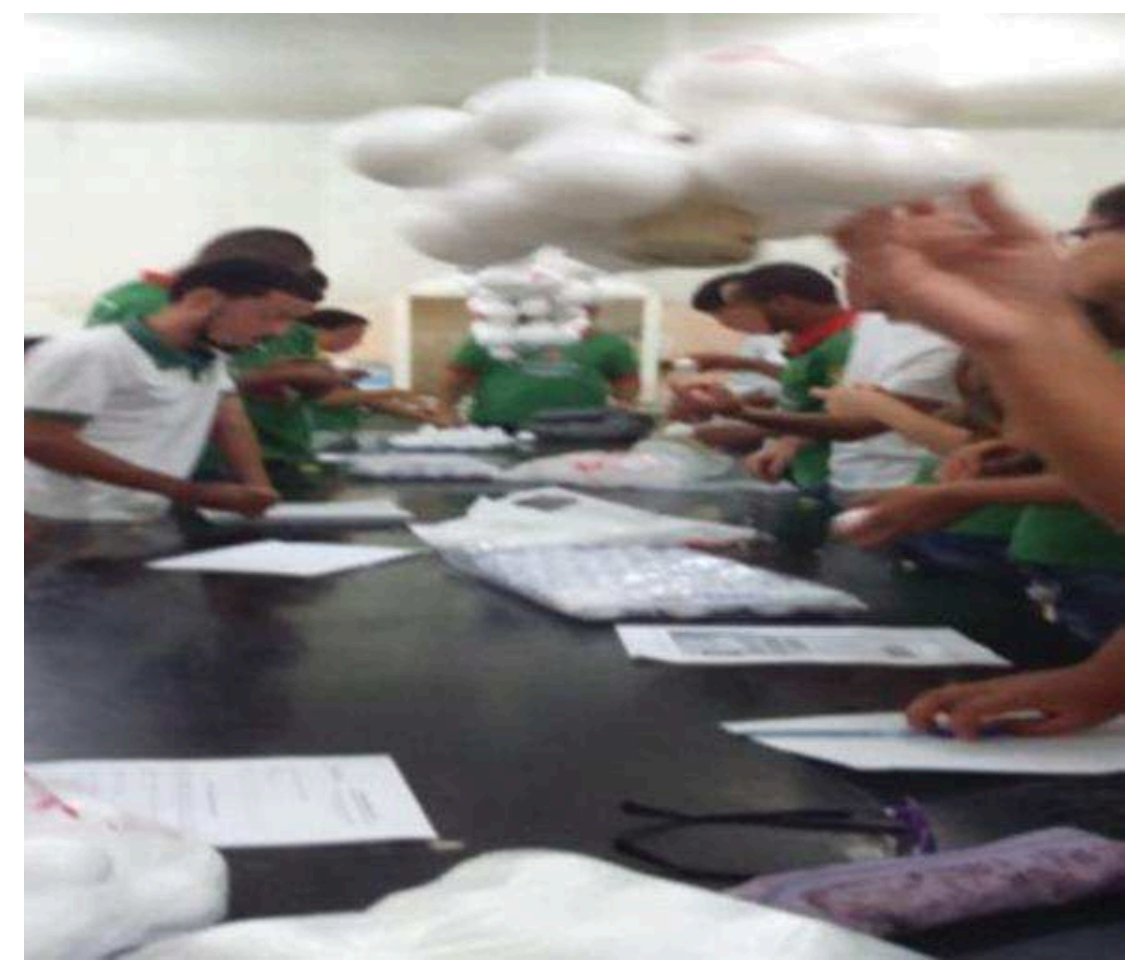

Fig. 8 - Atividade lúdica de instabilidade nuclear realizada pelos estudantes de TI. (BATISTA, 2015)

$\mathrm{Na}$ aula sete, a inclusão de novos parâmetros esteve relacionada com a mudança da atividade do "dominó radioativo" para "quebra-cabeça radioativa". Após os estudantes desenvolverem, principalmente, os conceitos de decaimentos radioativos alfa e beta, nas aulas anteriores, eles tinham que montar o quebra-cabeça e fazer um relatório explicando os conceitos utilizados (Fig. 9).

Para finalizar o processo de "avaliação interna" da sequência de ensinoaprendizagem, os estudantes foram levados e responder o questionário 2 envolvendo situaçõesproblemas (BARRAGÁN et al., 2009) acerca dos conceitos de radiação, irradiação, contaminação radioativa, exposição, emissão e absorção da radiação (Fig. 10). 


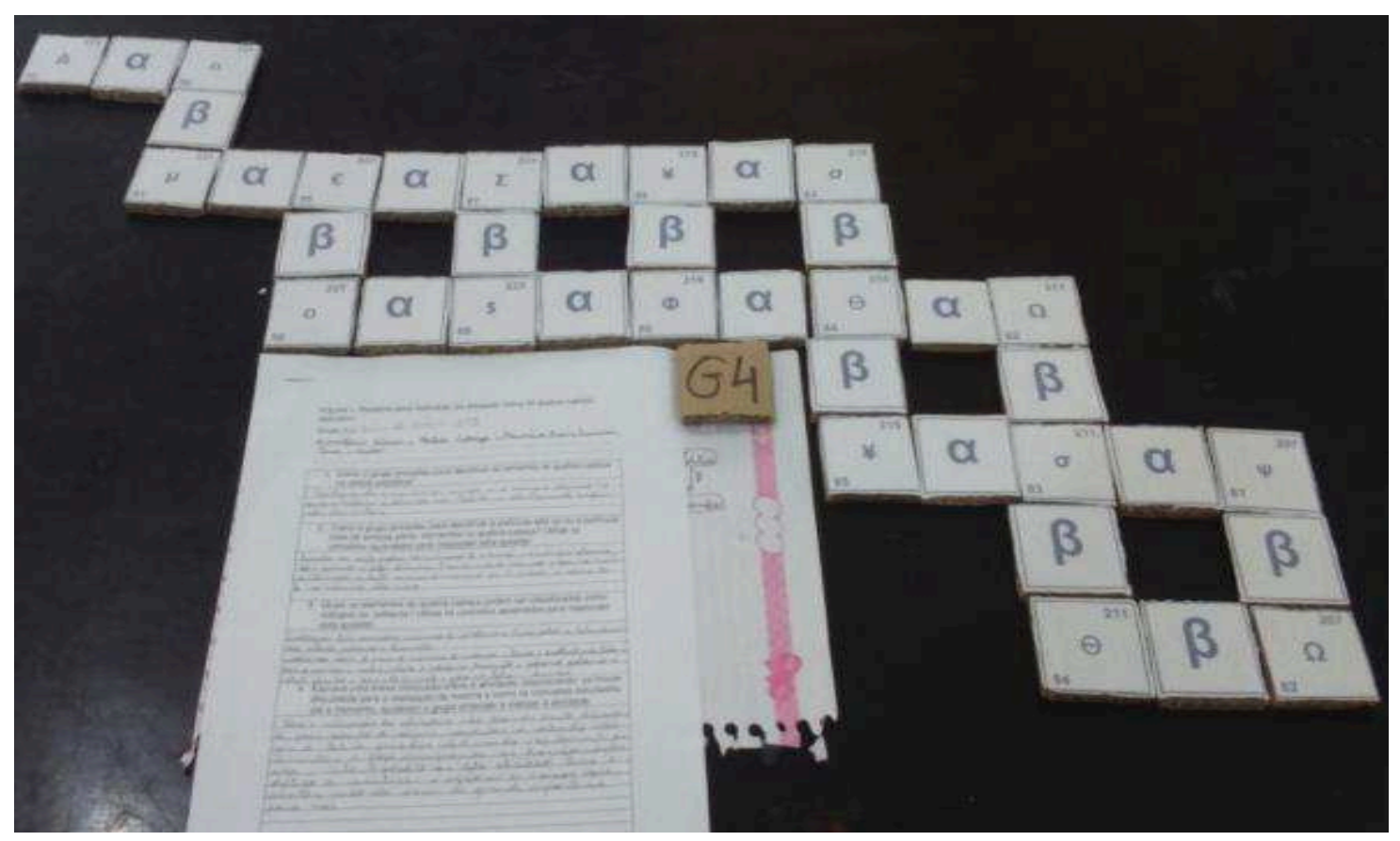

Fig. 9 - Atividade do quebra-cabeça radioativa montado pelos estudantes de TI. (BATISTA, 2015)

\begin{tabular}{|c|c|c|c|c|}
\hline \multirow{2}{*}{ Questões } & \multirow{2}{*}{ Sim } & \multirow{2}{*}{ Não } & \multicolumn{2}{|c|}{ Respostas } \\
\cline { 3 - 5 } & & & Não respondeu (*) & Outra resposta (\#). \\
\cline { 3 - 5 } & & & $2 *$ \\
\hline Q1 & 17 & 2 & $2^{*}$ \\
\hline Q2 & 17 & 4 & $2^{*}$ \\
\hline Q3 & 16 & 1 & $1^{*}$ \\
\hline Q4 & 16 & 3 & $7 \#$ \\
\hline Q6 & 9 & 11 & $4^{*}$ \\
\hline Q7 & 13 & 1 & $5^{*}$ \\
\hline Q8 & 14 & 3 & 16 & \\
\hline
\end{tabular}

Fig. 10 - Padrão de respostas apresentadas pelos estudantes de TI. (BATISTA, 2015)

Nossa fonte de dados constituiu-se de oito (8) questões respondidas por vinte e um (21) estudantes (168 respostas), que também revelaram a existência de um padrão de respostas às questões correlacionadas conceitualmente. Por exemplo, a Questão (1):

"ALIMENTOS IRRADIADOS são alimentos submetidos à radiação com o objetivo de reduzir a quantidade de microrganismos (como fungos e bactérias), presentes naturalmente, sem a necessidade de aditivos químicos. Esse processo também aumenta o período de validade dos produtos, pois retarda o amadurecimento. A irradiação de alimentos não altera o gosto deles nem suas propriedades. A OMS (Organização Mundial da Saúde) confirma que esses 
produtos não oferecem riscos à saúde e não contém níveis de radiação acima do normal. Todos os alimentos irradiados devem, obrigatoriamente, apresentar essa informação no rótulo. Você consumiria um alimento irradiado? JUSTIFIQUE,"

Questão (2): "Considerando que o Brasil desperdiça milhões de toneladas de alimento em um ano, porque eles apodrecem, ou foram contaminados por microrganismos ou agentes químicos. IMAGINE a seguinte situação: Foi proposto ao governo que utilizasse em grande escala a tecnologia de IRRADIAÇÃO DE ALIMENTOS com o objetivo de diminuir essas perdas e aumentar as possibilidades de exportação, levando a um maior aproveitamento da produção brasileira. IMAGINE que esse projeto esteja em votação, que você é um membro do governo e o SEU voto será definitivo. Como você votaria?”. JUSTIFIQUE.

As respostas dos estudantes foram: Estudantes (E3-TI)-(Q1). "Sim. Pois a OMS confirma que esses alimentos não apresentam riscos a saúde." Estudantes (E3-TI)(Q2). "Votaria sim, como a própria OMS avisou, os produtos irradiados não oferecem riscos a saúde, com isso, ajudaria na conservação dos alimentos". Estudante (E6-TI)-(Q1). "Sim, consumiria já que essa organização confirma que esses produtos não oferecem riscos a saúde e obrigatoriamente vai ter essa informação no rótulo". Estudante (E6-TI)-(Q2). "Eu aprovaria, segundo a condição de ter um órgão que afirmou que eles não causam qualquer tipo de risco à saúde". Estudante (E10-TI)-(Q1). "Sim, eu comeria um alimento irradiado, pois não oferece risco a saúde e a quantidade de radiação é baixa e é utilizada para reduzir a quantidade de fungos e bactérias". Estudante (E10-TI)- (Q2). "Sim, pois através da irradiação muitos alimentos terão uma durabilidade elevada e com isso iria evitar o desperdício”.

Analisando as respostas, a explicação conceitual sobre irradiação e contaminação radioativa dada pelos estudantes, estão compatíveis com definição de Okuno (1988, p.20) em seu livro - Radiação, Efeitos, Ricos e Benefícios - utilizado como fonte bibliográfica durante as aulas.

Os alimentos irradiados, para fins de esterilização, com radiação gama emitida por uma fonte de Césio-137 ou Cobalto-60, não se tornam radioativos. Porém, os alimentos contaminados pela poeira radioativa, ou aqueles que receberam nutrientes radioativos via raiz, são radioativos (OKUNO, 1988, p. 20).

Do exposto, percebemos que, nos exemplos dados, os estudantes tiveram um posicionamento, frente à situação-problema, amparado por informações do texto, cujos conteúdos souberam interpretar e julgar. Essa atitude revelou que os mesmos conseguem fazer uma distinção importante entre irradiação e contaminação radioativa. Esse indicativo concreto do estreitamento das concepções prévias dos estudantes com os conceitos da radioatividade mostra a potencialidade da sequência de ensino-aprendizagem como meio de favorecimento para a inserção da FMC nas salas de aula da Educação Básica. Todavia, para os estudantes que responderam não (Fig. 10, coluna 3), demonstraram que entendem a irradiação 
como um processo que torna os produtos radioativos. Nessa mesma linha de raciocínio, Barragán et al. (2009) mostraram que em seus estudos preliminares acerca desses conceitos, $11 \%$ dos estudantes de três turmas do Ensino Fundamental de Belo Horizonte tiveram o mesmo posicionamento diante dessas duas situações-problemas. Para esses autores, "mesmo tendo as informações disponíveis no enunciado, os (11\%) estudantes, (assim como os nossos) pareceram inseguros quanto ao processo e suas possíveis consequências (BARRAGÁN et al., 2009, p. 6). Com efeito, a importância desses resultados se revela à medida que a sequência de ensino-aprendizagem é capaz de gerar conhecimentos compatíveis com os apresentados pela literatura, bem como mostrar-se, mais uma vez, como caminho essencial para transformar as demandas teórica da inovação e atualização curricular, por meio da inserção da FMC, em intervenções práticas nos ambientes reais de sala de aula, visando, em especial, uma melhoria da aprendizagem científica dos estudantes.

\section{Considerações finais}

Consideramos que o relativo sucesso da sequência de ensino-aprendizagem reside na sua capacidade de transformar as demandas teóricas inerentes à inovação curricular e metodológica, por meio da inserção da FMC, em intervenções práticas de sala de aula. Neste sentido, as características da $D B R-T L S$ revelam a importância da fundamentação para atrelar o desenvolvimento deste objeto de intervenção $(T L S)$ às teorias educacionais, a partir de hipótese teóricas que emergem desse entrelaçamento, como apresentados por Nicolau et al. (2013) e por Lijsen e Klaassen (2004). Com efeito, o processo cíclico da DBR-TLS mostra-se bastante consistente para o campo de investigação da inserção da FMC, pois evidencia que o processo de avaliação, redesenho e nova implementação não acontecem no campo teórico, mas sim dentro de um processo empírico (aplicação em sala de aula) que permite uma reflexão crítica de suas finalidades em cada reestruturação. Acreditamos que, como fruto de uma pesquisa sistemática e atenta aos desafios para a inserção da FMC em sala de aula, a sequência de ensinoaprendizagem aqui apresentada possa ser utilizada por demais professores e pesquisadores no âmbito da educação brasileira e contribua expressivamente para difundir e consolidar esse tipo de pesquisa no Brasil. Além disso, que possa servir ao seu real objetivo: melhorar a aprendizagem científica dos estudantes e tornar as aulas de Física menos tradicionais.

\section{Referências}

BATISTA, C. Física Moderna e Contemporânea no Ensino Médio: subsídios teórico-metodológicos para a sobrevivência do tópico radioatividade em ambientes reais de sala de aula. 2015. 181 f. Dissertação (Mestrado em Educação em Ciências) - UESC, Ilhéus, BA. 
SIQUEIRA, M. Do visível ao indivisível: uma proposta de Física de Partículas Elementares para o Ensino Médio. 2006. 193 f. Dissertação (Mestrado em Educação em Ciências) - Instituto de Física/Instituto de Química /Faculdade de Educação, USP, São Paulo.

BACHELARD, G. A formação do espírito científico: contribuição para uma psicanálise do conhecimento. Tradução: Estela dos Santos Abreu. 1. ed. Rio de Janeiro: Contraponto Editora, 1938. 316 p. v.1.

BARRAGÁN, P.; MORTIMER, E. F.; LEAL, A. Avaliação Preliminar sobre o Conceito de Radiação e algumas de suas Tecnologias: Ideias informais de estudantes do ensino médio. In: ENCONTRO NACIONAL DE PESQUISA EM EDUCAÇÃO EM CIÊNCIAS, 7, 2009. Santa Catarina. Atas... Florianópolis: ABRAPEC, 2009.

BENARROCH, B. A. El desarrollo cognoscitivo de los estudiantes en el área de la naturaleza corpuscular de la materia. Enseñanza de las Ciencias. Barcelona, v. 18, n. 2, p. 235-246, set. 2000 .

BENARROCH, B. A. Una Interpretación del desarrollo cognoscitivo de los alumnos en el área de la naturaleza corpuscular de la materia. Enseñanza de las Ciencias. Barcelona, v. 19, n. 1, p. 123-134, set. 2001.

BROUSSEAU, G. O não dito é essencial. Revista Nova Escola. Edição 264, 2013.

BROUSSEAU, N.; BROUSSEAU, G. Rationnels et décimaux dans la scolarité obligatoire. IREM de Bourdeaux, 1987.

CARMO, M. P.; MARCONDES, M. E. R.; MARTORANO, S. A. A. Uma interpretação da evolução conceitual dos estudantes sobre o conceito de solução e processo de dissolução. Revista Electrónica de Enseñanza de las Ciencias, Buenos Aires, v. 9, n. 1, p. 35-52, jun. 2010.

CHEVALLARD, Y. La Transposicion Didactica: Del saber sabio al saber enseñado.1. ed. Argentina: La Pensée Sauvage, 1991. 189p

COLLECTIVE. Design-Based Research: An emerging paradigm for educational inquiry. Educational Researcher. London, v. 32, n. 5, p. 1-5, feb. 2003.

COLLINS, A.; JOSEPH, D.; BIELACZYC, K. Design Research: Theoretical and Methodological Issues. The Journal of Learning Sciences. London, v. 13, n. 1, p. 15-42, dec. 2004.

DElizoicov, D.; ANGOTTI, J. A. P.; PERnAMBUCO, M. M. Ensino de ciências: Fundamentos e Métodos. 4. ed. São Paulo: Editora Cortez, 2002. 364p.

GIL, D. P.; SENENT, F.; SOLBES, J. La introducción a la Física Moderna: um ejemplo paradigmatico de cambio conceptual. Enseñanza de las Ciencias. Barcelona, v. 1, n. Extra, p. 209-210, set. 1987. 
LEACH, J.; SCOTT, P. Designing and evaluating science teaching sequences: an approach drawing upon the concept of learning demand and a social constructivist perspective on learning. Studies in Science Education. Bristol, v. 38, n. 1, p. 115-142, apr. 2002.

LIJSEN, P.; KLAASSEN, C. W. J. M. Didactical structures as an outcome of research on teaching-learning sequences? International Journal of Science Education. London, v. 26, n. 5, p. 537-554, apr. 2004.

LOPES, J. B. et al. Transversal traits in science education research relevant for teaching and research: A meta-interpretative study. Journal of Research in Science Teaching. Lyon, v. 47, n. 5, p. 574-599, apr. 2008.

MARTINS, R. A. Como Becquerel não descobriu a Radioatividade. Caderno Catarinense do Ensino de Física. Florianópolis, v. 7, n. 29, p. 27-45, mar. 1990.

MÉHEUT, M.; PSILLOS, D. Teaching-learning sequences: Aims and tools for science education research. International Journal of Science Education. London v. 26, n. 5, p. 515- 535, apr. 2004.

MONTEIRO, M. A.; NARDI, R.; BASTOS FILHO, J. B. Física Moderna e Contemporânea no ensino médio e a formação de professores: desencontros com a ação comunicativa e a ação dialógica emancipatória. Revista Electrónica de Investigación en Educación en Ciencias, Buenos Aires, v. 8, n. 1, p. 1-13, jun. 2012.

MOREIRA, M. A. Aprendizagem Significativa: a teoria e textos complementares. 1. ed. São Paulo: Editora Livraria da Física, 2011. 179p.

NETO, J. S.; CAVALCANTI, C. J. H.; OSTERMANN, F. Estratégias discursivas adotadas por professores em formação na compreensão do fenômeno da complementaridade em atividades didáticas mediadas pelo interferômetro virtual de Mach-Zehnder. Revista Brasileira de Pesquisa em Educação em Ciências. São Paulo, v. 15, n. 2, p. 293-320, mai/ago. 2015.

NICOLAU, J.; GURGEL, I.; PIETROCOLA, M. Estrutura Baseada em Fluxo: Sequência de Ensino-Aprendizagem Sobre Relatividade do Tempo. In: ENCONTRO NACIONAL DE PESQUISA EM EDUCAÇÃO EM CIÊNCIAS, 9, 2013, São Paulo, 2. Atas... Águas de Lindóia: ABRAPEC, 2013.

OKUNO, E. Radiação: Efeitos, Riscos e Benefícios. 2. ed. São Paulo: Editora HARBRAS, 1988. 81p.

OSTERMANN, F.; MOREIRA, M. A. Uma revisão bibliográfica sobre a área de pesquisa "física moderna e contemporânea no ensino médio". Investigação em Ensino de Ciência. Porto Alegre. v. 5, n. 1, p. 23-48, abr. 2000. 
PEREIRA. P. A.; OSTERMANN, F. Sobre o ensino de física moderna e contemporânea: uma revisão da produção acadêmica recente. Investigação em Ensino de Ciências. Porto Alegre, v. 14, n. 3, p. 393-420, abr. 2009.

PIETROCOLA, M. et al. Física em contextos: pessoal, social e histórico. Livro do Professor. 1. ed. São Paulo: FTD Editora, 2010. p. 528. v.3.

SANTOS, S. E.; PÉREZ-ESTEBAN, J. Estudiando el fenómeno de la radiactividad a través de noticias de prensa: el caso del espía russo envenenado. Revista Eureka sobre Enseñanza y Divulgación de las Ciencias. Cádiz, v. 9, n. 2, p. 294-306, jan. 2012.

TIBERGHIEN, A.; BARBOUX, M. Les reprèsentations des èlèves de sixième et leur èvolution tempèrature-chaleur. Revue Française de Pédagogie. Paris, v. 72, p. 71-86, dec.1983.

TIBERGHIEN, A. Construction of prototypical situations in teaching the concept of energy. In G. Welford, J. Osborne and P. Scott (eds.) Research in Science Education in Europe. CurrentIssues and Themes (London: Falmer Press), p. 100-114, 1996.

TIBERGHIEN, A.; VINCE, J.; GAIDIOZ, P. Design-based Research: Case of a teaching sequence on mechanics. International Journal of Science Education. London, v. 31, n. 17, p. 2275-2314, apr. 2009.

WU, Y.; TSAI, C. High School Students: Informal Reasoning on a Socio-scientific Issue: qualitative and quantitative analyses. International Journal of Science Education. London, v. 29, n. 9, p.1163-1187, dec. 2007.

YANG, F.; ANDERSON, O. R. Senior high school students' preference and reasoning modes about nuclear energy use. International Journal of Science Education. London, v. 25, n. 2, p. 221-244, dec. 2003. 